\title{
Modification of a High Frequency Oscillator Circuit With a Heated Expiratory Filter to Prevent Infectious Pathogen Transmission: A Bench Study
}

\author{
Shari I McKeown RRT MA and Nicola Serreqi RRT
}

\begin{abstract}
BACKGROUND: High frequency oscillation is a safe and effective treatment for patients with ARDS, but poses a patient and caregiver risk when the circuit is disconnected. We modified the circuit to include a heated expiratory filter, eliminating the need for daily filter changes due to buildup of condensate. The purpose of the study was to determine if substitution of the filter resulted in a clinically important change in delivered tidal volume or amplitude. We additionally compared expiratory resistance and measured efficacy for the substituted filter. METHODS: This bench study measured tidal volume and amplitude using 5 of each filter type across 6 patient setting scenarios. Filter efficacy was tested through an independent laboratory, and expiratory resistance measurements were taken after prolonged use with humidification. RESULTS: The clinically important threshold value for tidal volume (defined as $5 \%$ difference) was excluded by the limits of agreement, confirming that use of the modified circuit does not result in alterations in tidal volume. The clinically important threshold for amplitude (defined as $10 \%$ difference) was the same as the lower confidence interval on the lower limit of agreement, indicating it is possible for amplitude values to be different between the 2 filters. Filter efficacy for the substituted filter was not affected. Expiratory resistance was unchanged in the substituted filter, but nearly doubled for the manufacturer's filter after 48 hours. CONCLUSIONS: Modifying the circuit to include a heated expiratory filter does not affect tidal volume, and the filter material remains efficacious during oscillation. Amplitude varies under some conditions. Preventing the need for daily filter changes reduces the risk of alveolar de-recruitment. This does not completely eliminate exposure to expired gases, but provides an additional layer of protection against occupational exposure and nosocomial spread of respiratory pathogens. Further testing in a clinical environment is necessary. Key words: high frequency oscillation ventilation; infection control; critical care; respiratory therapy; filtration; pandemics; severe acute respiratory syndrome; SARS; influenza; airborne particulate matter; ARDS. [Respir Care 2012;57(4):531-536. (C) 2012 Daedalus Enterprises]
\end{abstract}

\section{Introduction}

High frequency oscillation (HFO) is safe and effective in improving oxygenation for patients with ARDS,,$^{1,2}$ and

\footnotetext{
At the time of this research, Mrs McKeown was affiliated with the Department of Professional Practice, Vancouver General Hospital, GF Strong Rehabilitation Centre, George Pearson Centre, and University of British Columbia Hospital, Vancouver Coastal Health Authority, Vancouver, British Columbia, Canada. She is now affiliated with the British Columbia Patient Safety and Quality Council, Vancouver, British Columbia, Canada. Mr Serreqi is affiliated with the Department of Respiratory Services, Vancouver General Hospital, Vancouver, British Columbia, Canada.
}

Mrs McKeown and Mr Serreqi presented a version of this paper at the may reduce mortality rates in this critically ill patient population. ${ }^{3}$ Oscillation may be required when patients develop ARDS from potentially lethal and highly transmissible viruses such as novel influenza H1N1 (swine flu), influenza H5N1 (avian flu), or severe acute respiratory

27th Conference on High Frequency Ventilation of Infants, Children, and
Adults, held March 23-27, 2010, in Snowbird, Utah, for which it won the
18th annual Jimmy Schultz award, dedicated to improving patient care
through research and innovative use of respiratory care technology.

The authors have disclosed no conflicts of interest.

Correspondence: Shari I McKeown RRT MA.E-mail: sharimck@gmail.com.

DOI: 10.4187/respcare.01219 
syndrome (SARS). ${ }^{4,5}$ Occupational transmission to healthcare workers from patients with bacterial pneumonia has also been described during respiratory care procedures that occur at close proximity. ${ }^{6}$ When using a high frequency oscillator, expiratory gas is vented out relief valves directly into the atmosphere. Furthermore, the piston movement can lead to aerosolization of expired droplet particles. $^{7}$ This may increase the likelihood of airborne dispersion over longer distances. ${ }^{7,8}$

In 2003, with the virus responsible for causing an outbreak of SARS thought to be transmissible through the airborne route, ${ }^{9}$ some critical care units looked carefully at the possibility of occupational transmission and declined the use of HFO for SARS patients who developed ARDS. ${ }^{7,10}$ Similar concerns were raised during the H5N1 pandemic alert of 2007, prompting the World Health Organization to place high frequency oscillation on the list of "aerosol generating medical procedures," recommending use only within a negative pressure room where all healthcare workers were protected with gowns, gloves, eye protection, and N95 masks. ${ }^{11}$

See the Related Editorial on Page 657

In 2007, Viasys Healthcare (now CareFusion) released a filtered circuit for the SensorMedics 3100B (CareFusion, San Diego, California) high frequency oscillator in Canada. The filters are non-heated, and when used in a humidified circuit, collect condensate and become saturated with water, increasing expiratory resistance and affecting ventilating pressures. Thus, the manufacturer recommends changing the filters every 24 hours. Some researchers indicate circuit disconnects may increase the risk of ventilator-associated pneumonia, ${ }^{12}$ although there is little evidence to support this claim. ${ }^{13}$ However, not only does disconnecting the circuit result in lung de-recruitment, it poses a serious occupational and environmental health risk as healthcare providers and family members would be exposed to potentially highly transmissible organisms.

To prevent the need for filter changes, we modified the adult circuit of the oscillator to include a heated expiratory filter. The intent of using the external heater housing is to mitigate the buildup of condensate within the filter material, thereby eliminating the need for filter changes and circuit disconnection. The primary purpose of this study is to determine if substitution of this heated filter for the brand used in the manufacturer's circuit has any clinically important effects on delivered tidal volume or amplitude. The secondary purposes of the study are to determine if the efficacy of the heated filter is reduced when used during oscillation, and to document expiratory resistance in comparison with the manufacturer's filter when used in a heated humidified circuit.

\section{QUICK LOOK}

\section{Current knowledge}

High frequency oscillation requires the use of a proprietary circuit that does not include a heated expiratory filter. In patients with communicable respiratory disease this may present a risk to caregivers.

\section{What this paper contributes to our knowledge}

The addition of a heated expiratory filter to the high frequency oscillator circuit does not alter tidal volume in a lung model; however, amplitude can be reduced in some circumstances. The filtration efficacy of the heated filter is not adversely affected by prolonged exposure to high frequency oscillation.

\section{Methods}

\section{Circuit Modification}

We modified the circuit (flexible patient circuit with dual heated wire, Cardinal Health, Palm Springs, California) (Fig. 1) of the oscillator to include an OmniFilter Reusable Bacterial Filter (Puritan Bennett, Pleasanton, California), with filtration efficiency of $>99.97 \%$ of $0.3 \mathrm{mi}-$ cron particles (most penetrating particle size) (Fig. 2). The OmniFilter was housed within a heated device (VH-210 bacterial filter heater, 100-120 volts, 10 Watts, Vadi Medical Technology, Taoyuan, Taiwan), with preset nonadjustable temperatures of $55-70^{\circ} \mathrm{C}$. The VH-210 bacterial filter heater is composed of a plastic casing surrounding an aluminum sleeve with a coil heater. The bacterial filter heater and filter assembly were placed inline with the expiratory limb of the oscillator circuit, proximal to the high pressure relief valve and mean pressure control valve, to filter expired gases. Silastic $22 \mathrm{~mm}$ inner diameter connectors were used to connect the filter to the circuit without loss of circuit pressure, to avoid leaks, and to ensure a tight seal, avoiding risk of accidental disconnection.

This circuit modification resulted in an overall lengthening and sharper angle of the expiratory limb, contributing to an increased likelihood of expiratory tubing kinks proximal to the new filter. We added a short length of hard plastic splinting coil surrounding the tubing to mitigate this. This splinting reduced, but may not have completely eliminated, the incidence of circuit kinks across this portion. Kinks are a potential hazard for any ventilator circuit, and there is a need for practitioner vigilance in monitoring circuit integrity. The weight of the bacterial filter heater housing (total weight $=1.02$ $\mathrm{kg}$ with filter) was supported through the addition of a metal bracket manufactured by biomedical technicians within our healthcare technology management team. 


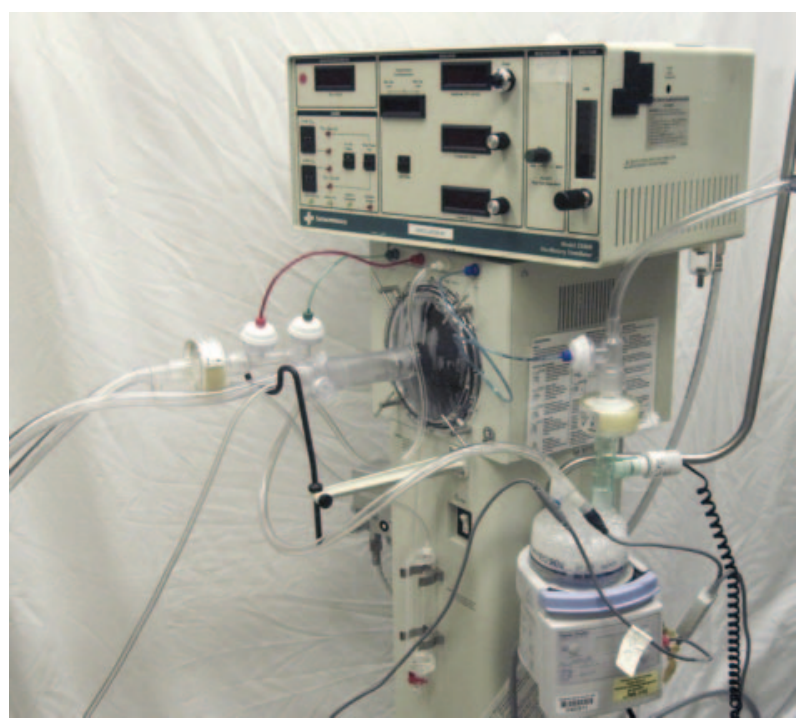

Fig. 1. SensorMedics 3100B original circuit. This photograph depicts the high frequency oscillator with the original circuit in place. Note the unheated AirLife filters present in the inspiratory and expiratory limbs of the circuit.

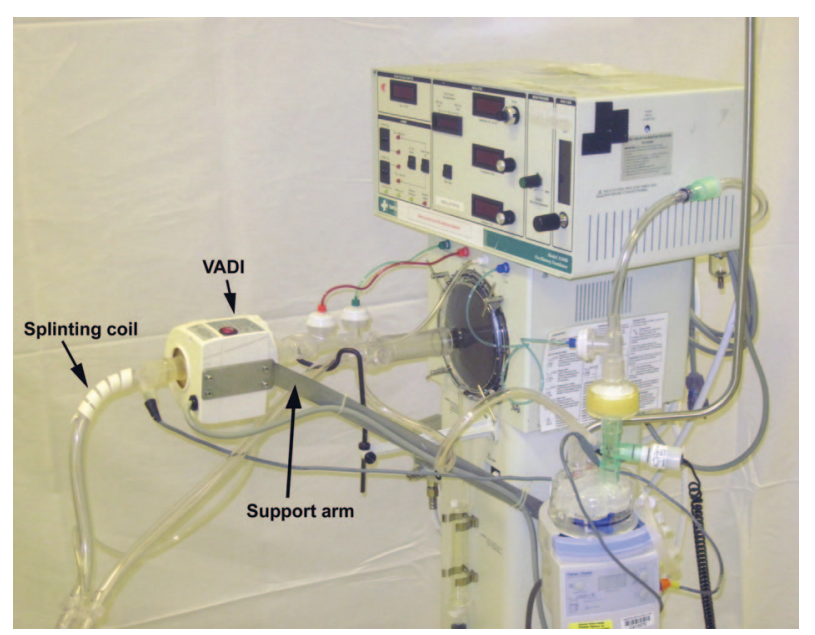

Fig. 2. SensorMedics 3100B circuit modification. The OmniFilter is housed within the heated bacterial filter heater assembly. A support arm attaches to the humidifier bracket and extends to the bacterial filter heater assembly, where it is screwed onto the side of the housing to support its weight. Hard plastic coil wrap is applied to splint the circuit where the tubing is most likely to kink in the expiratory limb.

\section{Circuit Modification Testing}

A patient circuit calibration test was performed on the modified circuit, and the results of the test fell within the manufacturer's specified range for pre-use testing. The patient Y-piece of the oscillator circuit was connected to the high flow oxygen inlet port of a VT Plus HF gas flow analyzer (Fluke Biomedical, Carson City, Nevada) using a single $15 \mathrm{~mm}$ outer diameter/15 mm inner diameter connector. A dual adult test lung (TTL 2600i, Michigan In-
Table 1. Patient Scenario Settings

\begin{tabular}{lcccc}
\hline \hline Scenario & $\begin{array}{c}\text { Mean Airway } \\
\text { Pressure } \\
\left(\mathrm{cm} \mathrm{H}_{2} \mathrm{O}\right)\end{array}$ & $\begin{array}{c}\text { Bias Flow } \\
\text { Rate } \\
(\mathrm{L} / \mathrm{min})\end{array}$ & Power & $\begin{array}{c}\text { Frequency } \\
(\mathrm{Hz})\end{array}$ \\
\hline 1 & 20 & 20 & 6 & 8 \\
2 & 25 & 30 & 7 & 8 \\
3 & 30 & 30 & 8 & 7 \\
4 & 35 & 30 & 9 & 6 \\
5 & 40 & 30 & 10 & 5 \\
6 & 45 & 40 & 10 & 4 \\
\hline
\end{tabular}

struments, Grand Rapids, Michigan), set with a compliance of $0.3 \mathrm{~L} / \mathrm{cm} \mathrm{H}_{2} \mathrm{O}$ and a resistance of $20.8 \mathrm{~cm} \mathrm{H}_{2} \mathrm{O}$ / $\mathrm{L} / \mathrm{s}$, was connected to the high flow exhaust port of the analyzer with a single $22 \mathrm{~mm}$ inner diameter connector.

The analyzer was used in HFO mode, in which it samples bidirectional flow and airway pressure at a rate 5 times greater than it does in normal operation (normal sample rate is $100 \mathrm{~Hz}$ ). Flow is measured via a differential pressure drop across a resistive screen mesh, and calibration of the device is traceable to the National Institute of Standards and Technology. The analyzer was zeroed prior to the first measurement and automatically throughout the data collection. Based on the analyzer manufacturer's recommendations and to ensure reliability in the comparison test, only dry gas was used during measurement. The relative humidity setting on the analyzer was set to ATPS (ambient temperature and pressure saturated) to optimize accuracy.

Measurements were taken using the nonheated expiratory filter included with the oscillator circuit (AirLife HEPA filter 001852, Allegiance Healthcare, McGaw Park, Illinois), and then compared to measurements taken using the OmniFilter (compliance $0.4 \mathrm{~mL} / \mathrm{cm} \mathrm{H}_{2} \mathrm{O}$ ). All other parts of the circuit remained the same for both measurements. Tidal volume and amplitude were measured using the analyzer value after allowing time for stabilization, with both circuit setups across 6 patient setting scenarios (Table 1). All measurements were repeated using 5 of each filter type, and mean values are reported.

\section{Filter Efficacy Testing}

The OmniFilter used in the modified circuit was not originally designed to be used with HFO. To ensure degradation of filtration material did not occur during use with the oscillator, we compared the efficacy of a new OmniFilter with that of a "test" (used) OmniFilter. The test filter was used within a closed heated humidified circuit for 9 continuous days of operation with the oscillator set to the following parameters: power 6.0 , percent inspiratory time $33 \%$, frequency $6 \mathrm{~Hz}$, bias flow $30 \mathrm{~L} / \mathrm{min}$, mean airway pressure $30 \mathrm{~cm} \mathrm{H}_{2} \mathrm{O}$.

The efficacy of the test filter was then compared to that of the new filter by an independent laboratory (Nelson 
Heated Expiratory Filter to Prevent Pathogen Transmission

Table 2. Amplitude and Tidal Volume for OmniFilter and AirLife Filter Across Patient Scenario Settings

\begin{tabular}{|c|c|c|c|c|c|c|c|}
\hline & \multirow[b]{2}{*}{ Filter } & \multicolumn{6}{|c|}{$\begin{array}{c}\text { Scenario } \\
(\text { mean } \pm \mathrm{SD})\end{array}$} \\
\hline & & 1 & 2 & 3 & 4 & 5 & 6 \\
\hline \multirow[t]{2}{*}{ Amplitude, $\mathrm{cm} \mathrm{H}_{2} \mathrm{O}$} & OmniFilter & $61.2 \pm 0.29$ & $70.3 \pm 0.23$ & $78.5 \pm 0.32$ & $90.5 \pm 0.95$ & $97.0 \pm 0.13$ & $95.0 \pm 0.21$ \\
\hline & AirLife & $66.5 \pm 0.22$ & $68.7 \pm 0.18$ & $79.9 \pm 0.29$ & $93.2 \pm 0.11$ & $102.8 \pm 0.39$ & $98.9 \pm 0.35$ \\
\hline \multirow[t]{2}{*}{ Tidal volume, $\mathrm{mL}$} & OmniFilter & $38.0 \pm 0.15$ & $40.3 \pm 0.08$ & $55.0 \pm 0.10$ & $69.0 \pm 0.26$ & $85.3 \pm 0.20$ & $101.9 \pm 0.21$ \\
\hline & AirLife & $38.5 \pm 0.14$ & $41.1 \pm 0.04$ & $55.9 \pm 0.11$ & $70.2 \pm 0.14$ & $86.9 \pm 0.10$ & $103.0 \pm 0.20$ \\
\hline
\end{tabular}

Laboratories, Salt Lake City, Utah). Both filters were subjected to a standardized sodium chloride aerosol efficiency test $^{14}$ to determine particle penetration and air flow resistance properties. A neutralized, poly-dispersed aerosol of sodium chloride, with a mass median diameter of approximately $0.26 \mu \mathrm{m}$ (accepted as the most penetrating particle size) was passed through the filters to calculate filtration efficiency.

\section{Filter Resistance Testing}

Filter resistance specifications for the AirLife filter were obtained from the manufacturer (testing per International Standards Organization standard 9360). These values were compared with the mean data from 3 Omnifilters, tested at a dry baseline, and after exposure to 24 and 48 hours of continuous HFO with heated and humidified gas within a closed circuit. OmniFilter resistance was measured by passing $100 \%$ oxygen gas through the filter at a flow rate of $60 \mathrm{~L} / \mathrm{min}$. Pressure measurements were taken at the inlet with the VT Plus HF gas flow analyzer, and values were recorded in $\mathrm{cm} \mathrm{H}_{2} \mathrm{O} / \mathrm{L} / \mathrm{s}$.

\section{Statistical Analysis}

We determined $95 \%$ limits of agreement ${ }^{15}$ by a linear mixed model ${ }^{16}$ that accounts for correlation between measurements made under the same scenario. The $95 \%$ limits of agreement provide an interval within which we would expect $95 \%$ of the differences between the 2 filters to lie. All statistical analyses were conducted using Stata 10 (StataCorp, College Station, Texas). A priori we defined absolute differences larger than $5 \%$ of the average tidal volume and $10 \%$ of the average amplitude to be clinically important.

\section{Results}

\section{Circuit Modification Testing}

The average mean tidal volume for all measurements with both filters was $65.9 \mathrm{~mL}$ (Table 2); thus, our threshold for agreement was $3.3 \mathrm{~mL}$. The estimated limits of agreement for tidal volume measurements were $-1.9 \mathrm{~mL}$

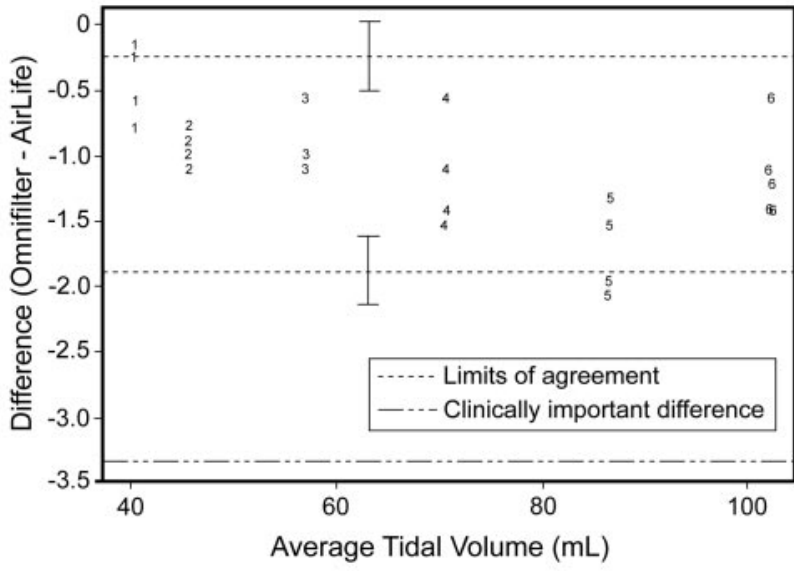

Fig. 3. Dot plot of differences between the 2 filters for tidal volume against their mean. The marker labels (1, 2, 3, 4, 5, and 6) indicates the patient scenario settings (see Table 1). Limits of agreement are designated as dotted lines, with $\mathrm{Cl}$ bars. The limits of agreement identify the interval in which $95 \%$ of the differences between filter measurements would be expected to lie. The level at which the differences would be considered clinically important is shown by the dotted-and-dashed line.

to $-0.24 \mathrm{~mL}$, with a margin of error of $\pm 0.3 \mathrm{~mL}$. (Fig. 3 ). Even allowing for sampling variability, the resulting range of $-2.2 \mathrm{~mL}$ to $0.06 \mathrm{~mL}$ still excludes the clinically important threshold value of $3.3 \mathrm{~mL}$. Thus, changing to the modified circuit did not result in clinically important differences in delivered tidal volumes when oscillator parameter settings are held constant. Amplitude measurements had a wider variation, and when assessing for clinically important measurements within $10 \%$ (agreement threshold $=8.4 \mathrm{~cm} \mathrm{H}_{2} \mathrm{O}$ ), this threshold was excluded by the estimated limits of agreement $\left(-7.2 \mathrm{~cm} \quad \mathrm{H}_{2} \mathrm{O}\right.$ to $0.49 \mathrm{~cm} \mathrm{H}_{2} \mathrm{O}$ ), but not when considering the margin of error for these estimates $\left( \pm 1.2 \mathrm{~cm} \mathrm{H}_{2} \mathrm{O}\right.$ ) (Fig. 4).

\section{Filter Efficacy Testing}

Filter testing results showed filtration efficiency at $99.778 \%$ for the used OmniFilter, when compared with a new filter of the same brand (filtration efficiency 99.53\%). 


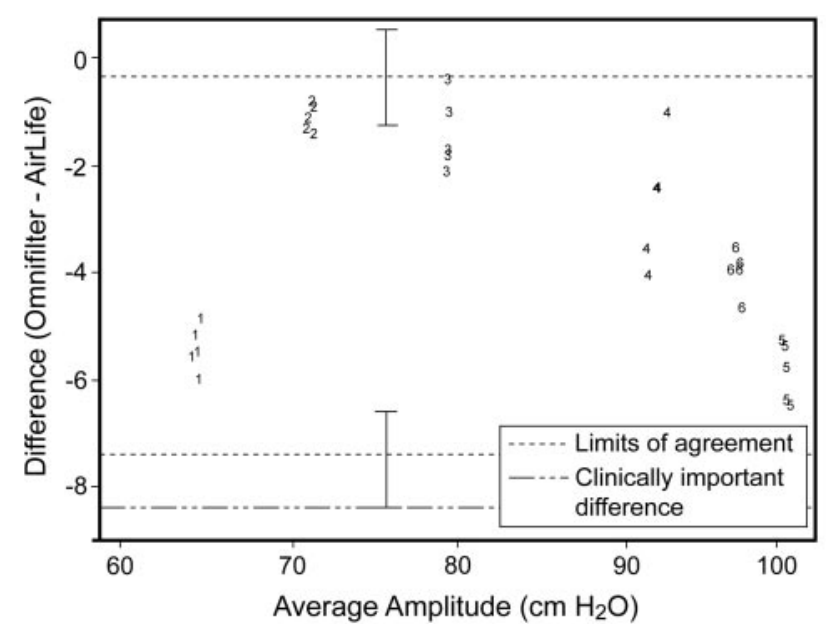

Fig. 4. Dot plot of differences between the 2 filters for amplitude against their mean. The marker labels $(1,2,3,4,5$, and 6$)$ indicates the patient scenario settings (see Table 1). Limits of agreement are designated as dotted lines, with $\mathrm{Cl}$ bars. The limits of agreement identify the interval in which $95 \%$ of the differences between filter measurements would be expected to lie. The level at which the differences would be considered clinically important is shown by the dotted-and-dashed line.

\section{Filter Resistance Testing}

The manufacturer's reported resistance measurement for the AirLife filter was $0.9 \mathrm{~cm} \mathrm{H}_{2} \mathrm{O} / \mathrm{L} / \mathrm{s}$ dry, $1.2 \mathrm{~cm} \mathrm{H}_{2} \mathrm{O} / \mathrm{L} / \mathrm{s}$ after 24 hours, and $1.7 \mathrm{~cm} \mathrm{H}_{2} \mathrm{O} / \mathrm{L} / \mathrm{s}$ after 48 hours. Mean measured expiratory resistance for the heated OmniFilter was $0.2 \mathrm{~cm} \mathrm{H} \mathrm{H}_{2} \mathrm{O} / \mathrm{L} / \mathrm{s}$ dry, $0.3 \mathrm{~cm} \mathrm{H}_{2} \mathrm{O} / \mathrm{L} / \mathrm{s}$ after 24 hours, and $0.3 \mathrm{~cm} \mathrm{H} \mathrm{H}_{2} \mathrm{O} / \mathrm{L} / \mathrm{s}$ after 48 hours.

\section{Discussion}

We have demonstrated that modifying the circuit of the SensorMedics 3100B high frequency oscillator to include a heated OmniFilter system in the expiratory limb does not have a clinically significant effect on the delivery of tidal volume within normal adult ARDS ranges of oscillation parameter settings when compared with the AirLife filter. Amplitude may show a clinically important difference with some settings, but in our bench study, these discrepancies did not change delivered tidal volume.

The OmniFilter has a larger compressible volume $(\sim 375 \mathrm{~mL})$ than the AirLife filter $(\sim 75 \mathrm{~mL})$. We noted a greater discrepancy in amplitude between the 2 filters in scenario 5 than in scenario 6 , despite the same power setting and lower mean airway pressure. We attribute this to the inability of the lower bias flow setting of $30 \mathrm{~L} / \mathrm{min}$ in scenario 5 to maintain amplitude with the higher dead space volume of the OmniFilter. When using the modified circuit on the highest power setting, bias flow rates may need to be increased to maintain the mean airway pressure at desired levels.
The VT Plus HF gas flow analyzer has accuracy specifications that may also account for some of the variation seen in amplitude measurements between the 2 filters tested. Pressure measurement accuracy is $\pm 0.8 \%$ of the reading, or $\pm 1.5 \mathrm{~mm} \mathrm{Hg}$, whichever is greater.

The expiratory resistance measurements with the heated Omnifilter stayed relatively constant from baseline, even after 48 hours exposure in a humidified circuit. In contrast, expiratory resistance specifications for the AirLife filter from dry baseline values almost double after 48 hours of use with humidity. A higher expiratory resistance in a high frequency oscillator circuit may result in a drift toward an increased mean airway pressure or amplitude; the clinical importance of this would be patient dependent.

The filtration components of the OmniFilter used in the circuit modification remain intact and efficacious after 9 days of continuous use; this filter is rated for up to a year of service or 100 autoclave cycles. The heated housing prevents the need for filter changes and resultant circuit disruptions. The manufacturer recommends oscillator circuits should be changed only as frequently as standard ventilator circuits as per an organization's policy. Preventing frequent circuit disruption protects patients against alveolar de-recruitment, may reduce the potential for introduction of infectious organisms, and lowers the risk of adverse events occurring during lung recruitment maneuvers that would need to be performed daily when using the AirLife filter.

This study was designed to compare 2 filters while holding constant other variables such as ventilation parameter settings, thoracic characteristics and physiology, endotracheal tube sizes and lengths, or other in vivo factors affecting system compliance or resistance. Measurements were taken with dry gas rather than with heated humidity, which would have more closely approximated clinical conditions. It is possible that results from this bench study will not translate into similar results when the circuit is used in a context with variable factors. However, in practice, most clinicians do not adopt a one-size-fits-all ventilation strategy, and instead titrate ventilation parameters to fit the needs of the patient. Therefore, we could speculate that any discrepancies in the pressure or volume outputs after circuit modification that were not accounted for in the in vitro design will have less of a clinical impact, as ventilation settings and adjustments are dynamic and are adjusted based on patient assessments and lab/radiographic results within a clinical environment.

It is important to note that any modification to a ventilator circuit carries inherent risk and is not recommended by the manufacturer. The modified circuit has not been tested in a clinical environment, and is therefore not recommended for use with humans. Clinical testing is necessary to ensure safety.

We modified and assessed an adult circuit only, as our facility does not provide care for neonatal or pediatric pop- 


\section{Heated Expiratory Filter to Prevent Pathogen Transmission}

ulations. Results should not be extrapolated to use in neonatal circuits or other models of high frequency oscillators; this warrants further study so that effective filtration can be applied for these patient populations. Clinicians should be aware that endotracheal tube cuff deflation techniques for carbon dioxide clearance negate the intent of expiratory gas filtration provided by the modified circuit. When endotracheal cuffs are inflated, air leaks are still possible and variable; clinicians should be aware of the inherent risk at all times, despite circuit filtration. An additional understanding of the limitations of all layers of protection (eg, accidental circuit disconnection, endotracheal dislodgement or cuff leak, improper use or fit of personal protective equipment, incomplete immunization, inadequate hand-washing) is essential to preventing infectious disease transmission; none of these strategies should be assumed fail-safe, and the addition of multiple layers of protection starting at the source, including use of filtration, may be employed as a comprehensive strategy to reduce infectious spread. ${ }^{5,17}$

\section{Conclusions}

Substituting a heated filter for the brand used in the manufacturer's circuit did not have any clinically important effects on delivered tidal volume during bench testing, although amplitude was affected under some parameter setting scenarios. The Omnifilter used in our modification remained efficacious after 9 continuous days of use. The heated housing surrounding the filter prevented buildup of condensate; expiratory resistance remained relatively constant, compared to the filter used in the manufacturer's circuit.

Removing the need for daily circuit disruption due to filter changes reduces the risk of alveolar de-recruitment. Expiratory filtration does not completely eliminate exposure to expired gases due to potential or purposeful leaks around the tracheal tube cuff. However, mitigating the need to open the circuit as frequently provides an additional layer of protection against occupational exposure and nosocomial spread of respiratory pathogens. Further safety testing in a clinical environment is necessary.

\section{ACKNOWLEDGMENTS}

The authors wish to thank Dr Penny Brasher, Biostatistician at the Centre for Clinical Epidemiology and Evaluation in Vancouver, British Columbia, for her assistance in calculating the limits of agreement.

\section{REFERENCES}

1. Chan KP, Stewart TE, Mehta S. High-frequency oscillatory ventilation for adult patients with ARDS. Chest 2007;131(6):1907-1916.
2. Derdak S, Mehta S, Stewart TE, Smith T, Rogers M, Buchman TG, et al. High frequency oscillatory ventilation for acute respiratory distress syndrome in adults: a randomized, controlled trial. Am J Respir Crit Care 2002;166(6):801-808.

3. Sud S, Sud M, Friedrich JO, Meade MO, Ferguson ND, Wunsch H, Adhikari NK. High frequency oscillation in patients with acute lung injury and acute respiratory distress syndrome (ARDS): systematic review and meta-analysis. BMJ 2010;340:c2327:1-11.

4. Kumar A, Zarychanski R, Pinto R, Cook DJ, Marshall J, Lacroix J, et al. Critically ill patients with 2009 influenza A (H1N1) infection in Canada. JAMA 2009;302(17):1872-1879.

5. Arabi Y, Gomersall CD, Ahmed QA, Boynton BR, Memish ZA. The critically ill avian influenza A (H5N1) patient. Crit Care Med 2007; 35(5):1397-1403.

6. Baba H, Iinuma Y, Imaizumi K, Hasegawa Y, Hasegawa Y, Ohta M, Paterson DL. Transmission of bacterial infections to healthcare workers during intubation and respiratory care of patients with severe pneumonia. Infect Cont Hosp Epidemiol 2009;30(10):1019-1021.

7. Sweeney A, Lyle J, Ferguson ND. Nursing and infection-control issues during high-frequency oscillatory ventilation. Crit Care Med 2005;33(Suppl 3):S204-S208.

8. Somogyi R, Vesely AE, Takafumi A, Preiss D, Fisher J, Correia J, Fowler RA. Dispersal of respiratory droplets with open vs closed oxygen delivery masks: implications for the transmission of severe acute respiratory syndrome. Chest 2004;125(3):1155-1157.

9. Yu ITS, Li Y, Tze Wai Wong, Tam W, Chan AT, Lee JHW, et al. Evidence of airborne transmission of the severe acute respiratory syndrome virus. N Engl J Med 2004;350(17):1731-1739.

10. Lapinsky SE, Hawryluck L. ICU management of severe acute respiratory syndrome. Intensive Care Med 2003;29(6):870-875.

11. World Health Organization Regional Office for the Western Pacific. Avian influenza, including influenza A (H5N1), in humans: WHO interim infection control guideline for health care facilities. May 10, 2007. http://www.wpro.who.int/internet/resources.ashx/CSR/ Publications/AI_Inf_Control_Guide_10May2007.pdf. Accessed January 30, 2012.

12. Fink JB, Krause SA, Barrett L, Schaaf D, Alex CG. Extending ventilator circuit change interval beyond 2 days reduces the likelihood of ventilator-associated pneumonia. Chest 1998;113(2):405-411.

13. Dodek P, Keenan S, Cook D, Heyland D, Jacka M, Hand L, et al. Evidence-based clinical practice guideline for the prevention of ventilator-associated pneumonia. Ann Intern Med 2004;141(4):305-313.

14. National Institute for Occupational Safety and Health (NIOSH). Respiratory protective devices. Code of Federal Regulations, Title 42, Part 84. Morgantown, WV: National Institute for Occupational Safety and Health; 1995.

15. Bland JM, Altman DG. Measuring agreement in method comparison studies. Stat Methods Med Res 1999;8(2):135-160.

16. Carstensen B, Simpson J, Gurrin LC. Statistical models for assessing agreement in method comparison studies with replicate measurements. Int J Biostat 2008;4(1):16.

17. Thiessen RJ. The impact of severe acute respiratory syndrome on the use of and requirements for filters in Canada. Respir Care Clin N Am 2006;12(2):287-306

This article is approved for Continuing Respiratory Care Education credit. For information and to obtain your CRCE

(free to AARC members) visit www.RCJournal.com

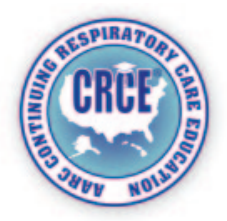

